\title{
A prominent role for amygdaloid complexes in the Variability in Heart Rate (VHR) during Rapid Eye Movement (REM) sleep relative to wakefulness
}

\author{
Martin Desseilles, ${ }^{\mathrm{a}, \mathrm{b}, *}$ Thanh Dang Vu, ${ }^{\mathrm{a}, \mathrm{c}}$ Steven Laureys, ${ }^{\mathrm{a}, \mathrm{c}}$ Philippe Peigneux, ${ }^{\mathrm{a}, \mathrm{d}}$ \\ Christian Degueldre, ${ }^{\mathrm{a}}$ Christophe Phillips, ${ }^{\mathrm{a}}$ and Pierre Maquet ${ }^{\mathrm{a}, \mathrm{c}}$ \\ ${ }^{\mathrm{a}}$ Cyclotron Research Centre, Liège University, Belgium \\ ${ }^{\mathrm{b}}$ Department of Psychiatry, CHU Liège, Belgium \\ ${ }^{\mathrm{c}}$ Department of Neurology, CHU Liège, Belgium \\ ${ }^{\mathrm{d}}$ Neuropsychology Unit, Liège University, Belgium
}

Received 3 October 2005; revised 31 May 2006; accepted 1 June 2006

Available online 27 July 2006

\begin{abstract}
Rapid eye movement sleep (REMS) is associated with intense neuronal activity, rapid eye movements, muscular atonia and dreaming. Another important feature in REMS is the instability in autonomic, especially in cardiovascular regulation. The neural mechanisms underpinning the variability in heart rate (VHR) during REMS are not known in detail, especially in humans. During wakefulness, the right insula has frequently been reported as involved in cardiovascular regulation but this might not be the case during REMS. We aimed at characterizing the neural correlates of VHR during REMS as compared to wakefulness and to slow wave sleep (SWS), the other main component of human sleep, in normal young adults, based on the statistical analysis of a set of $\mathrm{H}_{2}{ }^{15} \mathrm{O}$ positron emission tomography (PET) sleep data acquired during SWS, REMS and wakefulness. The results showed that VHR correlated more tightly during REMS than during wakefulness with the $\mathrm{rCBF}$ in the right amygdaloid complex. Moreover, we assessed whether functional relationships between amygdala and any brain area changed depending the state of vigilance. Only the activity within in the insula was found to covary with the amygdala, significantly more tightly during wakefulness than during REMS in relation to the VHR. The functional connectivity between the amygdala and the insular cortex, two brain areas involved in cardiovascular regulation, differs significantly in REMS as compared to wakefulness. This suggests a functional reorganization of central cardiovascular regulation during REMS.

(c) 2006 Elsevier Inc. All rights reserved.
\end{abstract}

Keywords: REM sleep; Heart rate; Functional neuroimaging; Positron emission tomography; Functional connectivity; Amygdala; Insula

\footnotetext{
* Corresponding author. Cyclotron Research Centre, Liège University, Belgium.

E-mail address: M.Desseilles@ulg.ac.be (M. Desseilles).

Available online on ScienceDirect (www.sciencedirect.com).
}

\section{Introduction}

Rapid eye movement sleep (REMS) is characterized by lowamplitude, relatively high-frequency electroencephalographic (EEG) rhythms, rapid eye movements and a complete muscular atonia interrupted by short muscular twitches. In addition, during REMS, neurovegetative regulation exhibits distinct features that are observed neither during wakefulness nor during non-REM sleep (NREMS). A striking example concerns thermoregulation. During REMS, a warm thermal load does not induce skin vasodilatation whereas a cold thermal load does not elicit any cutaneous vasoconstriction (Parmeggiani, 1980). These findings suggest that REMS is characterized by an "open-loop" mode of regulation, which does not rely on homeostatic feedback loops as strictly as during wakefulness or NREMS (Parmeggiani, 1985). During these 2 states, "closed-loop operations of automatic control mechanisms [...] warrant an efficient and steady regulation of [autonomic] functions" (Parmeggiani, 1985). These rules presumably apply also to other neurovegetative systems. Accordingly, respiratory and heart rates are known to be much more variable during REMS than during NREMS or wakefulness (Orem and Keeling, 1980).

Although cardiovascular regulation is understood in detail, the cerebral correlates of VHR have been characterized only recently, and exclusively during wakefulness. In humans, VHR has primarily been related to the activity in the insular cortex. Intraoperative electrical stimulation of the insula elicits changes in heart rate and blood pressure (Oppenheimer et al., 1992). In normal subjects, functional neuroimaging studies showed that in response to physical exercise (Williamson et al., 1997, 1999) and mental stressor tasks (Critchley et al., 2000), both associated with significantly increased heart rate, the activity in both insula covaried with heart rate.

Heart rate regulation changes during sleep and has also been related to forebrain activity, as assessed by EEG recordings. For 
instance, EEG power spectral density relates to VHR indices (Otzenberger et al., 1997, 1998; Ehrhart et al., 2000; Brandenberger et al., 2001; Ako et al., 2003). However, the cerebral correlates of VHR during sleep needs to be further characterized, anatomically refined and described separately for NREMS and REMS. Indeed, heart rate regulation differs between these 2 types of sleep due to predominant parasympathetic and sympathetic drives, respectively (Brandenberger, 2005). In this paper, we were particularly interested in characterizing the cerebral correlates of VHR during REMS because of the intriguing autonomic control described in this stage of sleep. We hypothesized that during REMS, heart rate regulation involves the amygdala. This structure is one of the most active brain areas during REMS in man (Maquet et al., 1996). Due to its anatomical connectivity, it is in good position to influence key regions involved in cardiovascular regulation like the hypothalamus and the parabrachial complex in the brainstem (Hopkins and Holstege, 1978). The paraventricular nucleus of the hypothalamus is a key site for regulating autonomic activities such as blood pressure and heart rate (Coote, 1995; Xia and Krukoff, 2003). The parabrachial complex is known to be implied in the regulation of sympathetic activity and heart rate (Henderson et al., 2002).

We examined the cerebral correlates of VHR in REMS, as compared to wakefulness and SWS, in humans, using positron emission tomography (PET). To do so, we conducted a retrospective analysis on a set of PET scans acquired in 13 non-sleep deprived normal participants during SWS, REMS or wakefulness with simultaneous electroencephalographic and electrocardiographic recordings. We determined the brain areas where the regional blood flow (CBF) was more tightly related to the VHR during REMS than during wakefulness, during SWS than during wakefulness or during SWS than during REMS. We focused on a set of target areas identified as critical in autonomous regulation during wakefulness: the insula (Cechetto and Saper, 1987; Oppenheimer et al., 1992; Oppenheimer, 1994; Corfield et al., 1995; Oppenheimer et al., 1996; Williamson et al., 1997; Critchley et al., 2000), the amygdala (Orem and Keeling, 1980; Sei and Morita, 1996; Critchley et al., 2000), the hypothalamus (paraventricular nucleus) (Hopkins and Holstege, 1978; Coote, 1995; Xia and Krukoff, 2003) and the midbrain (Herbert et al., 1990; Chamberlin and Saper, 1992; Henderson et al., 2002). Other areas more occasionally implicated in heart rate regulation were also considered as potential regions of interest: the hippocampus (Rowe et al., 1999; Ribeiro et al., 2002; Pedemonte et al., 2003), the anterior cingulate cortex (Buchanan et al., 1985; Neafsey, 1990), the ventromedial prefrontal cortex (Buchanan et al., 1985; Neafsey, 1990), the motor cortex (Critchley et al., 2000), the neostriatum (Delgado, 1960; Bradley et al., 1987, 1991; Lin and Yang, 1994; Critchley et al., 2000), the cerebellum (Delgado, 1960; Bradley et al., 1987, 1991; Lin and Yang, 1994; Critchley et al., 2000) and the brainstem areas of the pons and medulla (Willette et al., 1984; Allen and Cechetto, 1992; Critchley et al., 2000).

\section{Methods}

\section{Subjects and experimental protocol}

Data were obtained from previous sleep studies conducted in our center using the $\mathrm{H}_{2}{ }^{15} \mathrm{O}$ infusion method (Maquet et al., 2000; Peigneux et al., 2003). All subjects were young, healthy, righthanded and male volunteers ( $n=13$; age range $20-30$ years) who gave their informed consent to participate in studies approved by the Ethics Committee of the Faculty of Medicine of the University of Liège. All had normal sinus rhythm and regular sleep-wake habits. None had any medical, surgical or psychiatric history; none was taking medication. Each subject spent three consecutive nights in the PET scanner at usual sleep time. Polysomnography monitoring during the first two nights allowed us to check for any abnormality in sleep (insomnia, sleep fragmentation, REMS onset, etc.) and accustomed participants to the experimental setting. Participants were selected for the third night if they could maintain 20 min of continuous stage 2, stages 3-4 of NREMS and REMS on both habituation nights. During the third night, PET scans were performed both during various stages of sleep when polysomnography showed steady characteristic sleep patterns and during waking at rest with eyes closed in complete darkness. During waking scans, the subjects had to stay still, eyes closed.

At least two waking, two stage 2, two stages 3-4 and two REMS scans were obtained in all subjects. In the present manuscript, we used 97 PET scans (30 during W, 29 during SWS and 38 during REMS) from 13 subjects who all had high-quality electrocardiographic (EKG) recordings in all the 3 main states of vigilance (wakefulness, NREMS, REMS). The same subjects were used for the delta analysis published by Dang-Vu et al. (2005).

\section{Sleep analysis}

Polysomnography was performed with a Synamp (Neuroscan, NeuroSoft Inc.K, Sterling, Virginia) system at $500 \mathrm{~Hz}$ or $1000 \mathrm{~Hz}$, with a band width of $0.15-100 \mathrm{~Hz}$. EEG on (at least) C3-A2 and C4-A1 derivations were recorded. In all cases, vertical and horizontal electrooculograms, chin electromyographic derivation and chest electrocardiogram were recorded on bipolar montage. Sleep scoring followed standard international criteria (Rechtschaffen and Kales, 1968).

\section{Heart rate analysis}

The analysis was performed on the 90-s recordings obtained during each PET scan. The EKG was visually checked in order to discard any period containing movement, muscle or breathing artefact. A template of the QRS complex was generated by averaging the QRS complexes over the whole $90 \mathrm{~s}$ of recording. A coefficient of correlation was computed at each time point between the template and the actual recording using a sliding window. Correlation coefficient above 0.80 was shown to reliably identify the occurrence of a QRS complex. This threshold was used to detect $\mathrm{R}$ events. RR intervals, from these tagged events were then computed, generating a new time series covering the whole 90 -s scanning period. The variability in heart rate (VHR) was simply estimated as the standard deviation of the duration of RR intervals, as it has been described as a valid measure of the VHR (Malik, 1996).

\section{PET data acquisitions}

PET data were acquired on a Siemens CTI 951 R 16/31 scanner in three-dimensional mode. The head of the subjects was stabilized by a thermoplastic face mask secured to the head holder (Truscan Imaging, Annapolis, Maryland), and a venous catheter was inserted in a left antebrachial vein. First, a 20-min transmission scan was acquired for attenuation correction using three rotating 
sources of 68-Ge. Then, when polysomnography showed stable characteristic patterns, $\mathrm{rCBF}$, taken as a marker of local neuronal activity (Jueptner and Weiller, 1995), was qualitatively estimated during a maximum of 12 emission scans per subject using the $\mathrm{H}_{2}{ }^{15} \mathrm{O}$ technique. Each scan consisted of two frames: a $30-\mathrm{s}$ background frame and a 90-s acquisition frame. The slow intravenous water $\left(\mathrm{H}_{2}{ }^{15} \mathrm{O}\right)$ infusion began $10 \mathrm{~s}$ before the second frame. Six millicuries (mCi) equivalent to 222 megaBecquerel $(\mathrm{MBq})$ was injected for each scan, in 5 cubic centimeters (cc) saline, over a period of $20 \mathrm{~s}$, starting $10 \mathrm{~s}$ before the onsets of the active frame. The infusion was totally automated in order not to disturb the subject during the scanning period. Data were reconstructed using a Hanning filter (cutoff frequency: 0.5 cycle/ pixel) and corrected for attenuation and background activity.

\section{Brain imaging data analysis}

PET data were analyzed using Statistical Parametric Mapping (SPM99; Wellcome Department of Cognitive Neurology, Institute of Neurology, London, UK) implemented in MATLAB ${ }^{\circledR}$ (The MathWorks, Inc., Natick, Massachusetts). For each subject, all scans were realigned to the first scan. PET images were then normalized to a standard template within the Montreal Neurological Institute (MNI) space (Frackowiak et al., 1997). Finally, normalized PET images were smoothed using a Gaussian Kernel of 16 millimeters full width at half maximum.

Data were analyzed using a general linear model, in a single step conforming to a fixed effects analysis. This analysis does not allow to partition variance in within- and between-subject components. The results therefore do not pertain to the population at large but only to the studied sample.

The design matrix included 2 regressors: the main effect of the condition (state of vigilance: REMS, SWS or Wakefulness) and the condition (state of vigilance) by VHR interaction.

Global flow adjustment was performed by proportional scaling. Areas of significant changes were determined using linear contrasts. The contrast of interest estimated the condition and VHR effects, as well as the interactions between the condition and VHR (REMS versus SWS; SWS versus wakefulness; REMS versus wakefulness). Each of these contrasts identified the brain areas where the regional activity is more tightly related to VHR during the first than the second state of vigilance.

A psychophysiological interaction was also analyzed. This analysis assessed whether the relationship between the activity in the reference region identified (i.e., the amygdala, see Results section) and other distant areas depends on the state of vigilance (Wakefulness or REMS) (Friston et al., 1997). A new linear model was constructed using three regressors. The first regressor was the condition effect (REMS versus wakefulness). The second regressor was the activity in the reference area (amygdala, coordinates: 36,8 , $-20 \mathrm{~mm}$ ). The third regressor represented the interaction of interest between the first (psychological) and second (physiological) regressor.

In both analyses, the resulting set of voxel values for the contrast of interest constituted a map of the $t$ statistic $\{\mathrm{SPM}(\mathrm{T})\}$, thresholded at $p<0.001 \quad(Z \geq 3.09)$. Corrections for multiple comparisons were then performed at the voxel level over the entire brain volume or over small volumes (Small Volume Correction $\{\mathrm{SVC}\}$ with spheres of $10 \mathrm{~mm}$ ) centered on coordinates previously published in the literature (Critchley et al., 2002a, 2002b; see also introduction).

\section{Results}

\section{Statistical data}

A repeated measure ANOVA was conduced with stage of vigilance (SWS versus REMS versus wakefulness) as within-subject factor and VHR as dependent variable. Results showed a trend for a difference between conditions $(F(2,24)=2,4006, p=0.1121)$. Planned comparisons compared REMS with SWS, SWS with wakefulness and REMS with wakefulness. VHR tended to differ between REMS and SWS ( $p=0.1043$ ), as well as between SWS and wakefulness $(p=0.958025)$. The only significant difference was detected between REMS and wakefulness $(p=0.03371)$.

\section{Imaging data}

\section{Difference in the distribution of cerebral activity between wakefulness and REMS}

The main effect of condition (state of vigilance: wakefulness or REMS) is reported to establish the consistency of the present findings with previous studies (Maquet et al., 1996; Braun et al., 1997; Maquet, 2000; Maquet et al., 2000; Peigneux et al., 2003, 2004) and will not be discussed further.

Regional CBF was significantly larger during REMS than during wakefulness in the occipital area, in the lateral and mesiotemporal regions, in the anterior cingulate and in the precentral cortex (Table 1). Conversely, higher activity during wakefulness (versus REMS) was found bilaterally in the dorsolateral prefrontal and parietal cortices and in the posterior cingulate cortex (Table 2).

\section{Difference in the distribution of cerebral activity between wakefulness and SWS}

The main effect of condition (state of vigilance: wakefulness or SWS) is reported to establish the consistency of the present findings with previous studies (see, for instance, Maquet, 2000) and will not be discussed further.

Table 1

Increased brain activity during REMS compared to wakefulness

\begin{tabular}{lrlrll}
\hline Area & $x$ & $y$ & $z$ & $Z$ score & $P$ \\
\hline Left lingual gyrus & -28 & -64 & -2 & 7.25 & $<0.001$ \\
Right lingual gyrus & 28 & -62 & 0 & 6.98 & $<0.001$ \\
Left cuneus & -16 & -90 & 20 & 6.19 & $<0.001$ \\
Right cuneus & 6 & -82 & 16 & 6.12 & $<0.001$ \\
Left precuneus & -20 & -48 & 54 & 6.54 & $<0.001$ \\
Right anterior cingulate cortex & 6 & -2 & 40 & 6.34 & $<0.001$ \\
Left anterior cingulate cortex & -12 & -10 & 42 & 5.39 & 0.001 \\
Right medial temporal gyrus & 66 & -14 & -2 & 5.75 & $<0.001$ \\
Right superior temporal gyrus & -62 & -16 & 2 & 5.65 & $<0.001$ \\
Right precentral gyrus & 38 & -20 & 54 & 5.28 & 0.001 \\
Left amygdala & -30 & -2 & -18 & 4.50 & 0.042 \\
\hline Localzation & & & & &
\end{tabular}

Localization and statistical results concerning the local maxima of the brain areas where the activity is larger during REMS as compared to wakefulness. Coordinates are defined in the stereotatic MNI space, relative to anterior commissure. $x$ represents the lateral distance from midline (positive, right); $y$ is the anteroposterior distance from anterior commissure (positive: anterior); $z$ represents the rostrocaudal distance from the bicommissural plane (positive: rostral). These results survive a correction for multiple comparisons over the entire brain volume at a threshold of corrected $P<0.05$. 
Table 2

Increased brain activity during Wakefulness compared to REMS

\begin{tabular}{lrrrll}
\hline Area & \multicolumn{1}{l}{$y$} & $y$ & \multicolumn{1}{c}{$Z$} & $Z$ score & $P$ \\
\hline Right medial frontal gyrus & 44 & 50 & -2 & $>10$ & $<0.001$ \\
Right superior frontal gyrus & 42 & 52 & 8 & $>10$ & $<0.001$ \\
Right inferior frontal gyrus & 54 & 16 & 14 & 7.01 & $<0.001$ \\
Right precentral gyrus & 54 & 12 & 46 & 6.51 & $<0.001$ \\
Left medial frontal gyrus & -46 & 50 & 4 & $>10$ & $<0.001$ \\
Left inferior parietal gyrus & -44 & -56 & 38 & 6.14 & $<0.001$ \\
Left cingulate gyrus & -2 & -34 & 26 & 6.85 & $<0.001$ \\
\hline
\end{tabular}

Localization and statistical results concerning the local maxima of the brain areas where the activity is larger during wakefulness as compared to REMS. Coordinates and inferences are determined as in Table 1.

Regional CBF was significantly deactivated during SWS than during wakefulness in the thalami, orbital frontal cortex, precuneus and in the parietal cortex (Table 3).

\section{Effect of VHR on $C B F$}

We did not identify any area where the regional $\mathrm{CBF}$ was significantly related to the VHR irrespective of the state of vigilance (wakefulness or REMS or SWS).

\section{Condition (REMS versus Wakefulness) by VHR interaction}

The activity in the lateral aspect of the right amygdala (coordinates: $36,8,-20$ ) was shown to be related to VHR more tightly during REMS than in wakefulness $\left(Z=3.11, p_{\mathrm{SVC}}=0.028\right.$, reference coordinates taken in Critchley et al., 2002a, 2002b; Fig. 2). Due to the poor spatial resolution of PET scanning, it is not possible to further specify this area that probably encompasses part of the extended amygdala (Alheid and Heimer, 1988) (Fig. 1).

\section{Psychophysiological interaction}

Psychophysiological interaction assessed whether functional relationships between amygdala and any brain area were modulated by the state of vigilance. The psychophysiological interaction using the amygdala as reference region $(x=36, y=8, z=$ -20 ) identified a single area in the anterior insular cortex (Fig. 2A). This result indicates that the anterior insula is connected more tightly with the amygdala during wakefulness than during the REMS $\left(Z=3.11, p_{\mathrm{SVC}}=0.028\right.$, reference coordinates taken in Critchley et al., 2000) (Fig. 2B).

Insular cortex is involved in central processing of various sensory modalities. It is why we chose specifically for the small volume correction of our interest region the coordinates of a paper that investigates specifically the cardiovascular regulation to insure that the region is implicated in this physiological function (Critchley et al., 2000).

Table 3

Decreased brain activity during SWS compared to wakefulness

\begin{tabular}{lrrrll}
\hline Area & \multicolumn{1}{c}{$x$} & $y$ & \multicolumn{1}{l}{$z$} & $Z$ score & $P$ \\
\hline Orbital frontal cortex & 26 & 38 & -28 & $>10$ & $<0.001$ \\
Orbital frontal cortex & -22 & 40 & -26 & $>10$ & $<0.001$ \\
Left thalamus & -8 & 10 & -2 & $>10$ & $<0.001$ \\
Parietal cortex & 42 & -76 & 48 & 6.23 & $<0.001$ \\
Parietal cortex & -46 & -62 & 48 & 7.48 & $<0.001$ \\
Precuneus & -2 & -82 & 52 & 6.92 & $<0.001$ \\
\hline
\end{tabular}

Localization and statistical results concerning the local maxima of the brain areas where the activity is smaller during SWS as compared to wakefulness. Coordinates and inferences are determined as in Table 1.

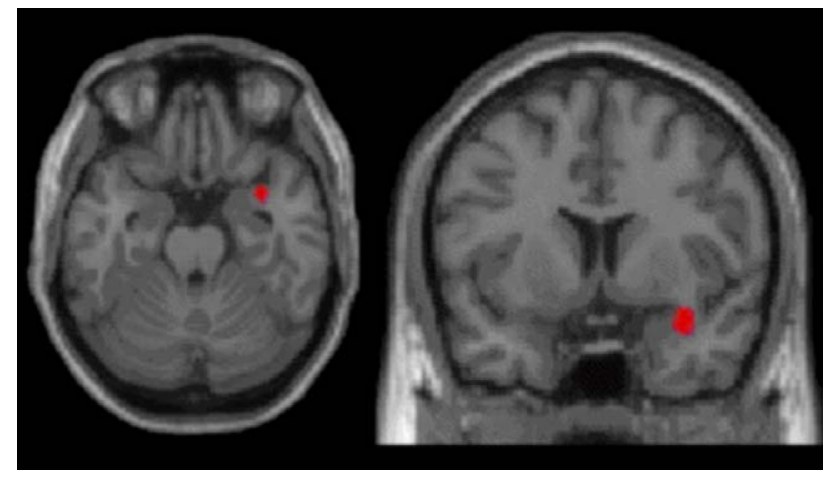

Fig. 1. The activity in the lateral aspect of the right amygdala (coordinates: $36,8,-20$ ) is more tightly related to VHR during REMS than during wakefulness $\left(Z=3.11, p_{\mathrm{SVC}}=0.028\right)$. The functional results are displayed over an individual MRI scan normalized to the MNI space in transverse (left panel), frontal (right panel) and sagittal planes. The functional results are displayed at $p<0.001$, uncorrected.

Condition (SWS versus wakefulness) by VHR interaction, condition (wakefulness versus SWS) by VHR interaction, condition (REMS versus SWS) by VHR interaction and condition (SWS versus REMS) by VHR interaction

These analyses did not identify a single significant change in regional cerebral blood flow $\left(p_{\text {uncorrected }}=0.001\right)$.

\section{Discussion}

\section{Methodological issues}

We used the standard deviation of heart rate as a measure of VHR during the 90-s duration of each PET scan. This parameter has been proposed as a valid estimation of VHR (Malik, 1996).

Alternatively, VHR can be estimated in the frequency domain. Two components can be isolated in a spectrum calculated from short-term recording: low-frequency (LF) and high-frequency (HF) components. The distribution of the power and the central frequency of LF and HF may vary in relation to changes in autonomous modulations of heart period (Malik, 1996). HF power would reflect parasympathetic activity, whereas LF power would primarily reflect a mixed sympathetic and parasympathetic influence (Malik, 1996). Due to methodological constraints of PET measurements, the time series recorded during the 90 -s scans were too short to obtain reliable heart rate power spectra. Recordings of approximately $1 \mathrm{~min}$ are needed to assess the high-frequency peak whereas at least 2 min recording are needed to reliably assess the lowfrequency component (Akselrod et al., 1981; Malik, 1996; Otzenberger et al., 1997, 1998; Brennan et al., 2002).

Consequently, we conservatively assessed VHR using the standard deviation of the heart rate. This measure is relatively more sensitive to high-frequency variations in heart rate (Malik, 1996).

It should be noted that a circadian effect is not likely to confound our results. The acquisition times for wakefulness, REMS and NREMS largely overlapped. Furthermore, a circadian factor is probably minor because VHR is highly sleep stage dependent (Zemaityte et al., 1984; Raetz et al., 1991; Vanoli et al., 1995; Pivik et al., 1996; Brandenberger et al., 2001; Viola et al., 2002). For instance, individual profiles revealed abrupt HR increases in each transition from deeper sleep to lighter sleep or awakening (Viola et al., 2002). The standard deviation of normal RR intervals and LF: 


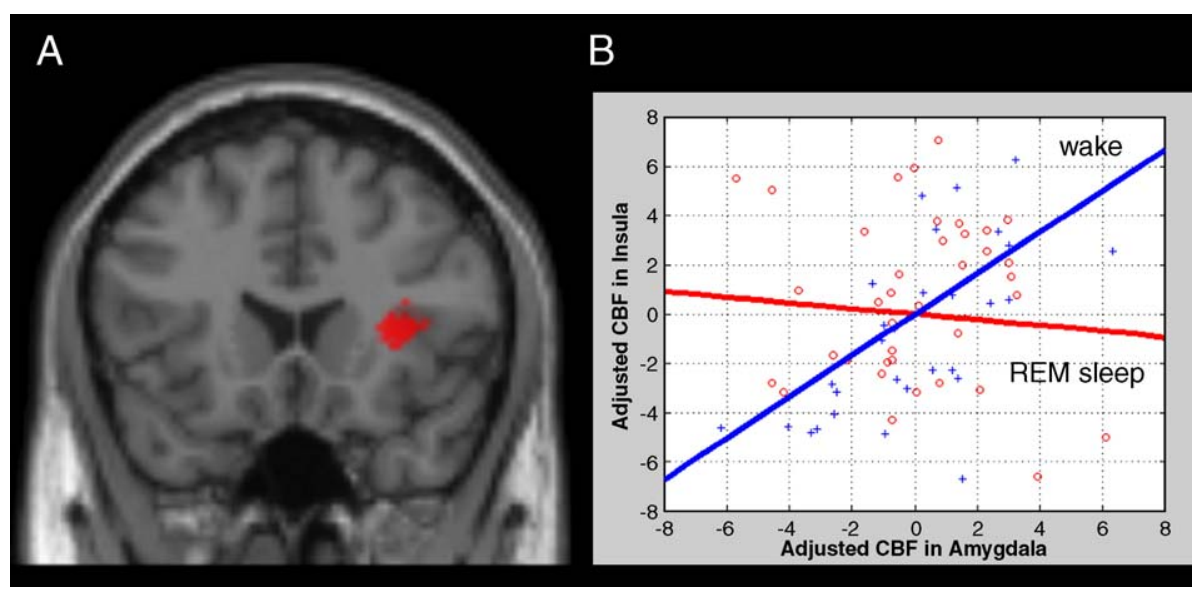

Fig. 2. (A) Psychophysiological interaction using the amygdala $\mathrm{rCBF}$ as reference region $(x=36, y=8, z=-20)$ and the state of vigilance (REMS versus wakefulness) as condition identified a single area in the right anterior insular cortex. The functional result is displayed over an individual MRI scan normalized to the MNI space in transverse, frontal and sagittal planes. The functional results are displayed at $p<0.001$, uncorrected. (B) Regression of insular on amygdalar activity during REMS and in wakefulness. This result indicates that the right anterior insula is connected more tightly with the amygdala during wakefulness than during the REMS $\left(Z=3.11, p_{\mathrm{SVC}}=0.028\right.$, reference coordinates taken in Critchley et al., 2000). The straight lines correspond to the regression, in wakefulness and REMS, respectively. The dots correspond to the adjusted rCBF observed in the 2 regions (red dots: REMS, blue cross: wakefulness).

$(\mathrm{LF}+\mathrm{HF})$ ratio decreases during SWS and significantly increases during REMS and during intrasleep awakening (Viola et al., 2002). However, the circadian factor cannot be totally ruled out. Although sympathetic nervous system activity is mostly influenced by sleep states, parasympathetic nervous system activity has been shown to be under circadian regulation (Burgess et al., 1997).

Dreams are known to occur frequently during REMS. One might argue that dream content would partly explain the variability in heart rate that ever report. As no dream reports were obtained from the subjects after each scan, we are in a position neither to confirm or falsify such a conclusion.

\section{Amygdala in the regulation of heart rate variability during REMS}

Central regulation of heart rate probably involves distributed networks encompassing cortical, hypothalamic and brainstem structures. At the cortical level, no significant effect of VHR was observed. This suggests that the functional neuroanatomy of central cardiovascular regulation varies between wakefulness, SWS and REMS. This hypothesis is further supported by the condition by VHR interaction.

The latter shows that the amygdala is the only area where the rCBF covaries with VHR differentially during REMS than during wakefulness. In particular, the activity in the amygdala is more tightly related to VHR during REMS than during wakefulness. No significant difference was observed between REMS and SWS and between SWS and wakefulness in terms of brain areas where the regional blood flow is related to VHR. Given results did not show any significant difference in VHR between SWS and wakefulness nor between SWS and REMS, we cannot argue that the modulatory influence of the amygdala is specific to REM sleep. However, we can suggest that the neural correlates of VHR differ between wakefulness and REM sleep. At present, no definite conclusion can be reached for SWS during which the cerebral correlates of VHR differed neither from wakefulness nor from REMS.

The coordinates of the significant changes in blood flow point to the lateral aspect of the amygdala. Although the poor spatial resolution of the PET cannot precisely describe the location of the amygdala response, it should be noted that these coordinates were reported in studies involving the right amygdala in cardiovascular regulation during wakefulness (Critchley et al., 2002a, 2002b).

The amygdala is intimately implicated in several basic features of REMS. In humans, the amygdala is particularly active in REMS (Maquet et al., 1996). In cats, cholinergic activation of the central amygdaloid nucleus produces a long-term facilitation of REMS occurrence (Calvo et al., 1996). Similarly, pharmacological stimulation (by vasointestinal peptide) of the amygdala induces increased amounts of REMS and ponto-geniculo-occipital waves (Simon-Arceo et al., 2003). Likewise, the rebound of REMS induced by microinjections of GABA agonist into the periaqueductal grey matter elicited a significant increase in c-fos labeling in the amygdala (Sastre et al., 2000).

Moreover, amygdala is in good position to influence critical regions for the cardiovascular regulation (Hopkins and Holstege, 1978), like the hypothalamus (Coote, 1995; Shannahoff-Khalsa and Yates, 2000; Xia and Krukoff, 2003) and parabrachial complex (Henderson et al., 2002).

The psychophysiological interaction also shows that the functional connectivity between amygdala and insula is different during REMS as compared to wakefulness. The functional relationship between the amygdalar and the insular cortices seems tighter during wakefulness than during REMS. During wakefulness, the insula plays a major role in cardiovascular regulation (Oppenheimer et al., 1992; Critchley et al., 2000). In normal humans, $\mathrm{rCBF}$ in the right insula covaries with heart rate (Critchley et al., 2000). Functional magnetic resonance imaging (fMRI) recently confirmed the functional links between the insular cortex and the modulation of heart rate (Williamson et al., 1997, 1999; Critchley et al., 2000). The activity in the left insula increases during dynamic exercise (cycling) but not passive exercise (cycling movement induced by moving pedals independently) (Williamson et al., 1997, 1999). Likewise, increased rCBF in right insula covaried with HR during isometric exercise and 
mental stressor tasks like arithmetic (Critchley et al., 2000). Moreover, the observations of brain damaged patients confirm the pivotal influence of the insular cortex in cardiovascular regulation (Oppenheimer, 1994; Oppenheimer et al., 1996; Tokgozoglu et al., 1999). The insular cortex is implicated in the generation of cardiac arrhythmias following hemispheric stroke (Oppenheimer, 1994). Accordingly, strokes in the region of insula (especially on the right side) leads to decrease VHR and to increased the incidence of sudden death (Tokgozoglu et al., 1999). Similarly, left-sided acute insular stroke "increase basal cardiac sympathetic tone and was associated with a decrease in randomness of heart rate variability" (Oppenheimer et al., 1996). In epileptic patients, electrical stimulations within the insula elicit changes in heart rate (Cechetto and Saper, 1987; Oppenheimer et al., 1992; Cechetto, 1994). Finally, it is known that the insula is anatomically and functionally connected with autonomic centers involved in heart rate regulation such as the amygdala and the hypothalamus (Augustine, 1996).

In contrast to what happens during wakefulness, our findings suggest that the amygdala largely influences heart rate during REMS. Moreover, our results suggest that this change in cardiovascular regulation is accompanied with a change in functional connectivity between the amygdala and the insula.

During REMS relative to wakefulness, the insula is less likely to modulate cardiovascular regulation, through its projections toward the amygdala. In this respect, the amygdala seems to take a prominent role in cardiovascular regulation during REMS in contrast to wakefulness. As amygdala is an integral part of the cerebral networks which generate and maintain REMS (Datta et al., 1998), the participation of the amygdala in VHR explains why the latter is an intrinsic characteristic of this sleep stage.

The modified cardiovascular regulation during REMS relative to wakefulness might have some bearing on important clinical issues. Many studies have demonstrated that the incidence of adverse cardiovascular events like sudden deaths or arrhythmias (Verrier et al., 1996) peak in the early morning hours (Muller et al., 1985, 1987; Tofler et al., 1987; Willich et al., 1987; Maron et al., 1994; Venditti et al., 1996; Elliott, 1998, 2001), and particularly during REMS (Schafer et al., 1997; Viola et al., 2002, 2004). Future research will have to assess the role of the amygdala and the change in its connectivity with the insular cortex in these lifethreatening events.

\section{Acknowledgments}

The authors thank the staff of the Cyclotron Research Centre for technical professional assistance. The study was supported by FNRS (Fonds National de la Recherche Scientifique), FMRE (Fondation Médicale Reine Elisabeth) and Research Fund of ULg. MD, TDV, PM and SL were supported by FNRS.

\section{References}

Ako, M., Kawara, T., Uchida, S., Miyazaki, S., Nishihara, K., Mukai, J., Hirao, K., Ako, J., Okubo, Y., 2003. Correlation between electroencephalography and heart rate variability during sleep. Psychiatry Clin. Neurosci. 57, 59-65.

Akselrod, S., Gordon, D., Ubel, F.A., Shannon, D.C., Berger, A.C., Cohen, R.J., 1981. Power spectrum analysis of heart rate fluctuation: a quantitative probe of beat-to-beat cardiovascular control. Science 213, $220-222$.

Alheid, G.F., Heimer, L., 1988. New perspectives in basal forebrain organization of special relevance for neuropsychiatric disorders: the striatopallidal, amygdaloid, and corticopetal components of substantia innominata. Neuroscience 27, 1-39.

Allen, G.V., Cechetto, D.F., 1992. Functional and anatomical organization of cardiovascular pressor and depressor sites in the lateral hypothalamic area: I. Descending projections. J. Comp. Neurol. 315, 313-332.

Augustine, J.R., 1996. Circuitry and functional aspects of the insular lobe in primates including humans. Brain Res. Brain Res. Rev. 22, 229-244.

Bradley, D.J., Paton, J.F., Spyer, K.M., 1987. Cardiovascular responses evoked from the fastigial region of the cerebellum in anaesthetized and decerebrate rabbits. J. Physiol. 392, 475-491.

Bradley, D.J., Ghelarducci, B., Spyer, K.M., 1991. The role of the posterior cerebellar vermis in cardiovascular control. Neurosci. Res. 12, 45-56.

Brandenberger, G., 2005. Endocrine correlates of sleep in humans. In: Parmeggiani, P.L., Velluti, R.A. (Eds.), The Physiologic Nature of Sleep. Imperial College Press, London.

Brandenberger, G., Ehrhart, J., Piquard, F., Simon, C., 2001. Inverse coupling between ultradian oscillations in delta wave activity and heart rate variability during sleep. Clin. Neurophysiol. 112, 992-996.

Braun, A.R., Balkin, T.J., Wesenten, N.J., Carson, R.E., Varga, M., Baldwin, P., Selbie, S., Belenky, G., Herscovitch, P., 1997. Regional cerebral blood flow throughout the sleep-wake cycle. An H2(15)O PET study. Brain 120 (Pt 7), 1173-1197.

Brennan, M., Palaniswami, M., Kamen, P., 2002. Poincare plot interpretation using a physiological model of HRV based on a network of oscillators. Am. J. Physiol.: Heart Circ. Physiol. 283, H1873-H1886.

Buchanan, S.L., Valentine, J., Powell, D.A., 1985. Autonomic responses are elicited by electrical stimulation of the medial but not lateral frontal cortex in rabbits. Behav. Brain Res. 18, 51-62.

Burgess, H.J., Trinder, J., Kim, Y., Luke, D., 1997. Sleep and circadian influences on cardiac autonomic nervous system activity. Am. J. Physiol. 273, H1761-H1768.

Calvo, J.M., Simon-Arceo, K., Fernandez-Mas, R., 1996. Prolonged enhancement of REM sleep produced by carbachol microinjection into the amygdala. NeuroReport 7, 577-580.

Cechetto, D.F., 1994. Identification of a cortical site for stress-induced cardiovascular dysfunction. Integr. Physiol. Behav. Sci. 29, 362-373.

Cechetto, D.F., Saper, C.B., 1987. Evidence for a viscerotopic sensory representation in the cortex and thalamus in the rat. J. Comp. Neurol. $262,27-45$.

Chamberlin, N.L., Saper, C.B., 1992. Topographic organization of cardiovascular responses to electrical and glutamate microstimulation of the parabrachial nucleus in the rat. J. Comp. Neurol. 326, 245-262.

Coote, J.H., 1995. Cardiovascular function of the paraventricular nucleus of the hypothalamus. Biol. Signals 4, 142-149.

Corfield, D.R., Fink, G.R., Ramsay, S.C., Murphy, K., Harty, H.R., Watson, J.D., Adams, L., Frackowiak, R.S., Guz, A., 1995. Evidence for limbic system activation during $\mathrm{CO}_{2}$-stimulated breathing in man. J. Physiol. 488 (Pt. 1), 77-84.

Critchley, H.D., Corfield, D.R., Chandler, M.P., Mathias, C.J., Dolan, R.J., 2000. Cerebral correlates of autonomic cardiovascular arousal: a functional neuroimaging investigation in humans. J. Physiol. 523 (Pt. 1), 259-270.

Critchley, H.D., Mathias, C.J., Dolan, R.J., 2002a. Fear conditioning in humans: the influence of awareness and autonomic arousal on functional neuroanatomy. Neuron 33, 653-663.

Critchley, H.D., Melmed, R.N., Featherstone, E., Mathias, C.J., Dolan, R.J., 2002b. Volitional control of autonomic arousal: a functional magnetic resonance study. NeuroImage 16, 909-919.

Dang-Vu, T.T., Desseilles, M., Laureys, S., Degueldre, C., Perrin, F., Phillips, C., Maquet, P., Peigneux, P., 2005. Cerebral correlates of delta waves during non-REM sleep revisited. NeuroImage 28, 14-21.

Datta, S., Siwek, D.F., Patterson, E.H., Cipolloni, P.B., 1998. Localization of pontine PGO wave generation sites and their anatomical projections in the rat. Synapse 30, 409-423.

Delgado, J.M., 1960. Circulatory effects of cortical stimulation. Physiol. Rev. 40 (Suppl 4), 146-178. 
Ehrhart, J., Toussaint, M., Simon, C., Gronfier, C., Luthringer, R., Brandenberger, G., 2000. Alpha activity and cardiac correlates: three types of relationships during nocturnal sleep. Clin. Neurophysiol. 111, 940-946.

Elliott, W.J., 1998. Circadian variation in the timing of stroke onset: a metaanalysis. Stroke 29, 992-996.

Elliot, W.J., 2001. Cyclic and circadian variations in cardiovascular events. Am. J. Hypertens. 14, 291S-295S.

Frackowiak, R., K. Friston, C. Frith, R. Dolan, J.C. Mazziotta, 1997. Human Brain Function. London.

Friston, K.J., Buechel, C., Fink, G.R., Morris, J., Rolls, E., Dolan, R.J., 1997. Psychophysiological and modulatory interactions in neuroimaging. NeuroImage 6, 218-229.

Henderson, L.A., Macey, P.M., Macey, K.E., Frysinger, R.C., Woo, M.A., Harper, R.K., Alger, J.R., Yan-Go, F.L., Harper, R.M., 2002. Brain responses associated with the Valsalva maneuver revealed by functional magnetic resonance imaging. J. Neurophysiol. 88, 3477-3486.

Herbert, H., Moga, M.M., Saper, C.B., 1990. Connections of the parabrachial nucleus with the nucleus of the solitary tract and the medullary reticular formation in the rat. J. Comp. Neurol. 293, 540-580.

Hopkins, D.A., Holstege, G., 1978. Amygdaloid projections to the mesencephalon, pons and medulla oblongata in the cat. Exp. Brain Res. 32, 529-547.

Jueptner, M., Weiller, C., 1995. Review: does measurement of regional cerebral blood flow reflect synaptic activity? Implications for PET and fMRI. NeuroImage 2, 148-156.

Lin, M.T., Yang, J.J., 1994. Stimulation of the nigrostriatal dopamine system produces hypertension and tachycardia in rats. Am. J. Physiol. 266, H2489-H2496.

Malik, M., 1996. Heart rate variability: standards of measurement, physiological interpretation and clinical use. Task Force of the European Society of Cardiology and the North American Society of Pacing and Electrophysiology. Circulation 93, 1043-1065.

Maquet, P., 2000. Functional neuroimaging of normal human sleep by positron emission tomography. J. Sleep Res. 9, 207-231.

Maquet, P., Peters, J., Aerts, J., Delfiore, G., Degueldre, C., Luxen, A., Franck, G., 1996. Functional neuroanatomy of human rapid-eyemovement sleep and dreaming. Nature 383, 163-166.

Maquet, P., Laureys, S., Peigneux, P., Fuchs, S., Petiau, C., Phillips, C., Aerts, J., Del Fiore, G., Degueldre, C., Meulemans, T., Luxen, A., Franck, G., Van Der Linden, M., Smith, C., Cleeremans, A., 2000. Experience-dependent changes in cerebral activation during human REM sleep. Nat. Neurosci. 3, 831-836.

Maron, B.J., Kogan, J., Proschan, M.A., Hecht, G.M., Roberts, W.C., 1994. Circadian variability in the occurrence of sudden cardiac death in patients with hypertrophic cardiomyopathy. J. Am. Coll. Cardiol. 23, 1405-1409.

Muller, J.E., Stone, P.H., Turi, Z.G., Rutherford, J.D., Czeisler, C.A., Parker, C., Poole, W.K., Passamani, E., Roberts, R., Robertson, T., et al., 1985. Circadian variation in the frequency of onset of acute myocardial infarction. N. Engl. J. Med. 313, 1315-1322.

Muller, JE., Ludmer, P.L., Willich, S.N., Tofler, G.H., Aylmer, G., Klangos, I., Stone, P.H., 1987. Circadian variation in the frequency of sudden cardiac death. Circulation 75, 131-138.

Neafsey, E.J., 1990. Prefrontal cortical control of the autonomic nervous system: anatomical and physiological observations. Prog. Brain Res. 85, 147-165; discussion 165-166

Oppenheimer, S.M., 1994. Neurogenic cardiac effects of cerebrovascular disease. Curr. Opin. Neurol. 7, 20-24.

Oppenheimer, S.M., Gelb, A., Girvin, J.P., Hachinski, V.C., 1992. Cardiovascular effects of human insular cortex stimulation. Neurology 42, 1727-1732.

Oppenheimer, S.M., Kedem, G., Martin, W.M., 1996. Left-insular cortex lesions perturb cardiac autonomic tone in humans. Clin. Auton. Res. 6, $131-140$.

Orem, Keeling, 1980. A compendium of physiology in sleep. In: Keeling, J.O.a.J. (Ed.), Physiology in Sleep. Academic Press, Lubbock, Texas.
Otzenberger, H., Simon, C., Gronfier, C., Brandenberger, G., 1997. Temporal relationship between dynamic heart rate variability and electroencephalographic activity during sleep in man. Neurosci. Lett. 229, 173-176.

Otzenberger, H., Gronfier, C., Simon, C., Charloux, A., Ehrhart, J., Piquard, F., Brandenberger, G., 1998. Dynamic heart rate variability: a tool for exploring sympathovagal balance continuously during sleep in men. Am. J. Physiol. 275, H946-H950.

Parmeggiani, 1980. Temperature regulation during sleep: a study in homeostasis. In: Keeling, J.O.a.J. (Ed.), Physiology in Sleep. Academic Press, Lubbock, Texas.

Parmeggiani, P.L., 1985. Regulation of circulation and breathing during sleep: experimental aspects. Ann. Clin. Res. 17, 185-189.

Pedemonte, M., Goldstein-Daruech, N., Velluti, R.A., 2003. Temporal correlations between heart rate, medullary units and hippocampal theta rhythm in anesthetized, sleeping and awake guinea pigs. Auton. Neurosci. 107, 99-104.

Peigneux, P., Laureys, S., Fuchs, S., Destrebecqz, A., Collette, F., Delbeuck, X., Phillips, C., Aerts, J., Del Fiore, G., Degueldre, C., Luxen, A., Cleeremans, A., Maquet, P., 2003. Learned material content and acquisition level modulate cerebral reactivation during posttraining rapid-eye-movements sleep. NeuroImage 20, 125-134.

Peigneux, P., Laureys, S., Fuchs, S., Collette, F., Perrin, F., Reggers, J., Phillips, C., Degueldre, C., Del Fiore, G., Aerts, J., Luxen, A., Maquet, P., 2004. Are spatial memories strengthened in the human hippocampus during slow wave sleep? Neuron 28, 535-545.

Pivik, R.T., Busby, K.A., Gill, E., Hunter, P., Nevins, R., 1996. Heart rate variations during sleep in preadolescents. Sleep 19, 117-135.

Raetz, S.L., Richard, C.A., Garfinkel, A., Harper, R.M., 1991. Dynamic characteristics of cardiac R-R intervals during sleep and waking states. Sleep 14, 526-533.

Rechtschaffen, A., Kales, A., 1968. A Manual of Standardized Terminology, Techniques and Scoring System for Sleep Stages of Human Subjects. National Institute of Health Publication, US Government Printing Office, Washington. DC.

Ribeiro, S., Mello, C.V., Velho, T., Gardner, T.J., Jarvis, E.D., Pavlides, C., 2002. Induction of hippocampal long-term potentiation during waking leads to increased extrahippocampal zif-268 expression during ensuing rapid-eye-movement sleep. J. Neurosci. 22, 10914-10923.

Rowe, K., Moreno, R., Lau, T.R., Wallooppillai, U., Nearing, B.D., Kocsis, B., Quattrochi, J., Hobson, J.A., Verrier, R.L., 1999. Heart rate surges during REM sleep are associated with theta rhythm and PGO activity in cats. Am. J. Physiol. 277, R843-R849.

Sastre, J.P., Buda, C., Lin, J.S., Jouvet, M., 2000. Differential c-fos expression in the rhinencephalon and striatum after enhanced sleepwake states in the cat. Eur. J. Neurosci. 12, 1397-1410.

Schafer, H., Koehler, U., Ploch, T., Peter, J.H., 1997. Sleep-related myocardial ischemia and sleep structure in patients with obstructive sleep apnea and coronary heart disease. Chest 111, 387-393.

Sei, H., Morita, Y., 1996. Acceleration of EEG theta wave precedes the phasic surge of arterial pressure during REM sleep in the rat. NeuroReport 7, 3059-3062.

Shannahoff-Khalsa, D.S., Yates, F.E., 2000. Ultradian sleep rhythms of lateral EEG, autonomic, and cardiovascular activity are coupled in humans. Int. J. Neurosci. 101, 21-43.

Simon-Arceo, K., Ramirez-Salado, I., Calvo, J.M., 2003. Long-lasting enhancement of rapid eye movement sleep and pontogeniculooccipital waves by vasoactive intestinal peptide microinjection into the amygdala temporal lobe. Sleep 26, 259-264.

Tofler, G.H., Brezinski, D., Schafer, A.I., Czeisler, C.A., Rutherford, J.D., Willich, S.N., Gleason, R.E., Williams, G.H., Muller, J.E., 1987. Concurrent morning increase in platelet aggregability and the risk of myocardial infarction and sudden cardiac death. N. Engl. J. Med. 316 , $1514-1518$.

Tokgozoglu, S.L., Batur, M.K., Top uoglu, M.A., Saribas, O., Kes, S., Oto, A., 1999. Effects of stroke localization on cardiac autonomic balance and sudden death. Stroke 30, 1307-1311. 
Vanoli, E., Adamson, P.B., Ba, L., Pinna, G.D., Lazzara, R., Orr, W.C., 1995. Heart rate variability during specific sleep stages. A comparison of healthy subjects with patients after myocardial infarction. Circulation 91 , 1918-1922.

Venditti Jr., F.J., John, R.M., Hull, M., Tofler, G.H., Shahian, D.M., Martin, D.T., 1996. Circadian variation in defibrillation energy requirements. Circulation 94, 1607-1612.

Verrier, R.L., Muller, J.E., Hobson, J.A., 1996. Sleep, dreams, and sudden death: the case for sleep as an autonomic stress test for the heart. Cardiovasc. Res. 31, 181-211.

Viola, A.U., Simon, C., Ehrhart, J., Geny, B., Piquard, F., Muzet, A., Brandenberger, G., 2002. Sleep processes exert a predominant influence on the 24-h profile of heart rate variability. J. Biol. Rhythms 17, 539-547.

Viola, A.U., Simon, C., Doutreleau, S., Ehrhart, J., Geny, B., Piquard, F., Brandenberger, G., 2004. Abnormal heart rate variability in a subject with second degree atrioventricular blocks during sleep. Clin. Neurophysiol. 115, 946-950.
Willette, R.N., Punnen, S., Krieger, A.J., Sapru, H.N., 1984. Interdependence of rostral and caudal ventrolateral medullary areas in the control of blood pressure. Brain Res. 321, 169-174.

Williamson, J.W., Nobrega, A.C., McColl, R., Mathews, D., Winchester, P., Friberg, L., Mitchell, J.H., 1997. Activation of the insular cortex during dynamic exercise in humans. J. Physiol. 503 (Pt 2), 277-283.

Williamson, J.W., McColl, R., Mathews, D., Ginsburg, M., Mitchell, J.H., 1999. Activation of the insular cortex is affected by the intensity of exercise. J. Appl. Physiol. 87, 1213-1219.

Willich, S.N., Levy, D., Rocco, M.B., Tofler, G.H., Stone, P.H., Muller, J.E., 1987. Circadian variation in the incidence of sudden cardiac death in the Framingham Heart Study population. Am. J. Cardiol. 60, 801-806.

Xia, Y., Krukoff, T.L., 2003. Differential neuronal activation in the hypothalamic paraventricular nucleus and autonomic/neuroendocrine responses to I.C.V. endotoxin. Neuroscience 121, 219-231.

Zemaityte, D., Varoneckas, G., Sokolov, E., 1984. Heart rhythm control during sleep. Psychophysiology 21, 279-289. 